\title{
Syntax score: The fallacies and remedies-A perspective
}

\author{
Pravin K. Goel ${ }^{*}$, Roopali Khanna, Aditya Batra, Sanjeev K. Syal \\ Department of Cardiology, Sanjay Gandhi Postgraduate Institute of Medical Sciences, Lucknow, India \\ Email: ${ }^{*}$ golf pgi@yahoo.co.in \\ Received 27 July 2013; revised 29 August 2013; accepted 12 September 2013 \\ Copyright (C) 2013 Pravin K. Goel et al. This is an open access article distributed under the Creative Commons Attribution License, \\ which permits unrestricted use, distribution, and reproduction in any medium, provided the original work is properly cited.
}

\begin{abstract}
The syntax score devised as a tool to grade complexity of coronary artery disease with a view to its suitability for PCI or otherwise on deeper introspection is fraught with some inherent limitations and fallacies largely as a result of the variability in coronary anatomy and the fixed nomenclature of the devised scoring system. We, in this special report, bring out the limitations and fallacies in the same and possible remedies presented as a viewpoint which may be useful to the interventional community at large.
\end{abstract}

Keywords: Syntax Score; PCI

\section{INTRODUCTION}

With advancements in percutaneous coronary intervention techniques and availability of reliable drug eluting stents, more and more patients with multivessel disease are being offered percutaneous coronary interventions (PCI). However, the best management strategy for an individual patient with multivessel disease, (i.e. either PCI or bypass surgery) has always been an issue of debate [1].

The SYNTAX score [2-4] was devised as an angiographic tool to grade the complexity of coronary artery disease (CAD) with a view to its suitability for PCI or otherwise. This score incorporates a combination of the effect of the area subtended by a diseased vessel/ segment in question, the severity of stenosis and the anatomical complexity of the lesion/lesions. It uses a combination of the AHA classification for coronary segments as modified for the ARTS study [5] and for the anatomical coronary segments, the Leaman score for weighted contribution of the stenosis to total myocardial jeopardy [6], the total occlusion classification system of the European Total Surveillance study [7] for classification of occlusions and the Duke and ICPS classification systems for

${ }^{*}$ Corresponding author. bifurcation lesions [8,9]. Although the syntax study was not meant to segregate patients based upon the Syntax score, it delivered an indirect message to the practising clinician and interventionists that if one could ascertain the syntax score in day to day practice and use the same in stratifying patients into those suitable for PCI or coronary artery bypass grafts (CABG), it may help in obtaining better clinical outcomes from the revascularization procedure.

Introspecting into the score, we have made some observations which we would like to bring to the attention of the cardiology community at large. These observations summarise the inherent fallacies/difficulties with the score and possible remedies, if any. The fallacies are largely a result of the high inter individual variability of the coronary anatomy and not uncommonly this variability may be so significant that the prefixed arterial nomenclature based scoring, as used by syntax, would not allow the flexibility needed for such wide variations in coronary anatomy. More so to have a computer generated software which would cover for all such variability would certainly not be easy. We have tried to work out the fallacies in some detail and the possible remedies in principle which could help make the score more precise and still maintain reproducibility.

\section{FALLACIES IN SYNTAX SCORE}

\subsection{Right Coronary Artery (RCA) vs Left Coronary Artery (LCA) Dominance Issue Incompletely Addressed}

The syntax scoring system accounts for right or left dominance based on Posterior Descending Artery (PDA) origin alone i.e. PDA from RCA or Circumflex $(\mathrm{Cx})$. However in real life extremes of dominance are not uncommon with a just dominant right system having PDA as the only off-shoot of RCA at one extreme (Figure 1(A)) and on the other the entire posterior and inferior Left Ventricular (LV) including the adjacent Obtuse Marginal $(\mathrm{OM})$ territory could be supplied by super domi- 
nant RCA (Figure 1(B)) while the usual average dominant RCA would encompass a PDA and at least one major adjacent posterior inferior LV branch (Figure 1(C)). It goes without saying then that all these three types of right dominance would call for the same myocardial weight of $1.0(16 \%$ of LV) in the syntax scoring system but in reality a lesion in each of these three different RCA's of same complexity should actually have a different clinical impact on total myocardial supply of the heart if left non revascularised. Similar logic could stand for variations in size of CX system (Figures 2(A) and (B)) independent of RCA dominance.

\subsection{LAD Length Issue Not Addressed at All}

The left anterior descending is known to vary in its length to a large extent and it is not uncommon to see patients with short LAD which does not even reach the apex (Type I) (Figure 3(A)) while there are others where the LAD is so long that it not only crosses the apex but also takes over almost the complete supply of PDA (Type III) and in these cases the PDA is invariably rather small

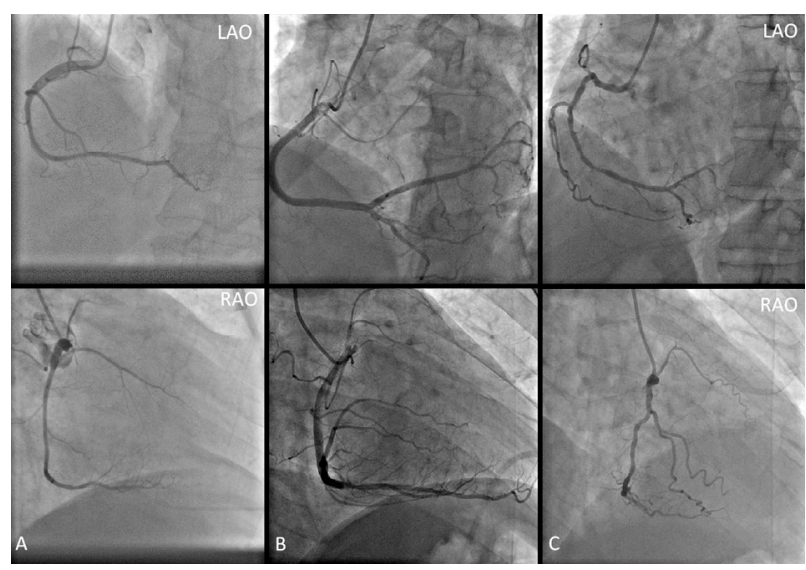

Figure 1. Dominant RCA of three varying sizes: (A) Just dominant RCA giving rise to PDA only; (B) Super-dominant RCA with PDA and 2/3 PLVs reaching the OM territory; (C) Average dominant RCA with PDA and one PLV.

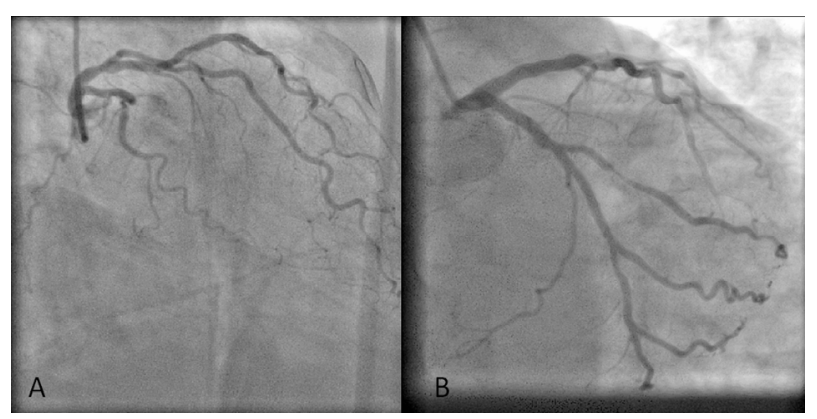

Figure 2. Both the angiograms show left injections from right dominant systems as per Syntax definitions with stark difference in size of LCX ((A): Small, (B): large) which can not be equated.

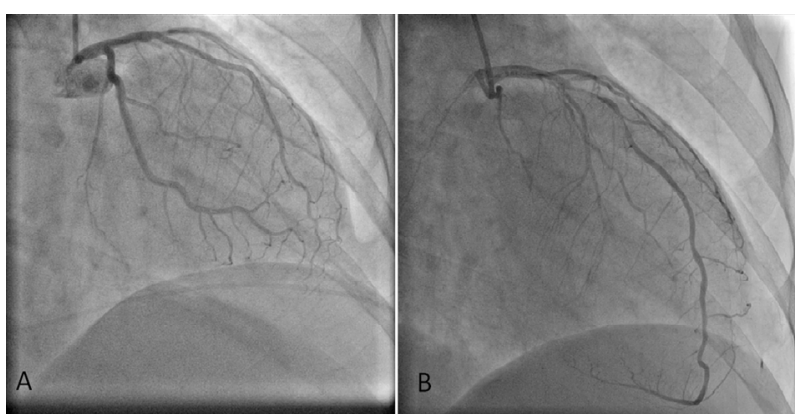

Figure 3. (A) LAD falling short of apex; (B) LAD wrapping around the apex and extending more than half the length into the posterior interventricular groove.

and of much smaller myocardial value (Figure 3(B)). It would then be quite unwise to give the same score to a lesion in mid or distal LAD in either of these two situations as advocated by the Syntax scoring system.

\subsection{Myocardial Weight of Proximal Segment Independent of the Distal Ramification of the Vessel}

If one takes the example of a RCA giving rise to a PDA (score 1.0) and two or three PLV's each being scored 0.5 respectively as per the syntax system then the total myocardial weight of this RCA becomes 2.5 but a lesion in the proximal RCA would continue to be weighed by a multiplication factor of 1.0 which is the fixed segmental weight of a dominant RCA main stem which does not sound to logic (Figure 4(A)). In the same context a proximal $\mathrm{Cx}$ in a usual right dominant circulation would continue to the weighed by a factor of 1.5 irrespective of the area of its distal supply with one, two or more OM's. Each of these individual OM's would add to the total weight of the vessel but a lesion in proximal or mid segment of the CX would continue to be weighted as per the fixed myocardial weight of proximal $\mathrm{Cx}$ of 1.5 as this is independent of the distal ramification of the vessel in the current syntax scoring system which seems incorrect.

\subsection{Score Dependent on the Nomenclature of the Vessel Rather than Its Anatomical Size}

The syntax score prefixes the myocardial weight of each individual branch of a coronary artery based on its nomenclature which by and large is reasonable but there are several situations involving vessels like the Ramus intermedius, OM and Diagonal (Dx) which at times are so huge that one single branch e.g. Ramus would cover the entire territorial supply (Figure 5(A)) of adjacent Dx and OM territories but would be equated to be of similar significance as any average size or even small ramus intermedius (Figure 5(B)) which by no means could equate to above. The only alternative is to exclude a particular 


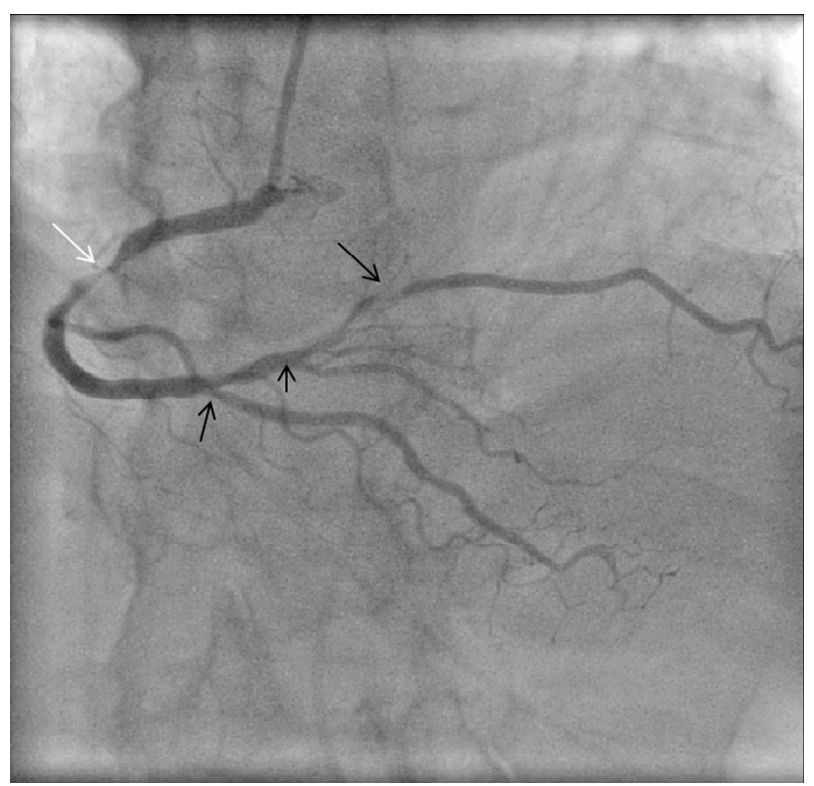

Figure 4. A-RCA has severe disease (white arrow) in proximal part which gets a value of only 1.0 in a right dominant system as per SYNTAX score but additional disease in PLV and PDA (black arrows) keeps adding a value of 0.5 each (total 1.5) making total weight of the vessel 2.5 but the proximal RCA lesion shall have myocardial value only 1.0 .

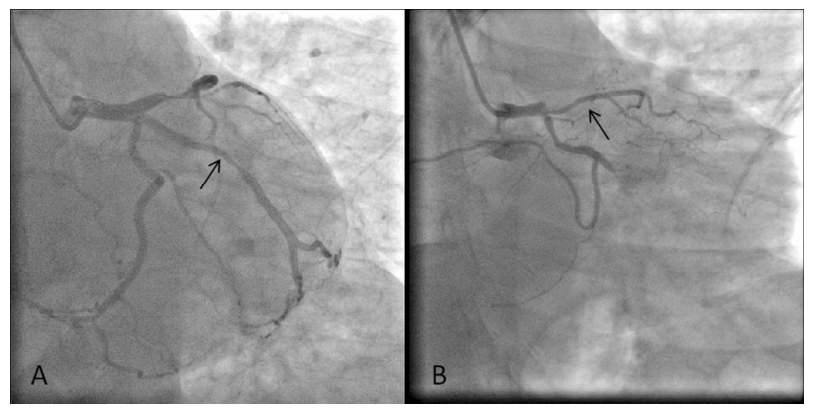

Figure 5. Two Ramus intermedius vessels ((A): small, (B): large) of contrastingly different sizes but getting the same value in SYNTAX scoring system since both are at least $1.5 \mathrm{~mm}$ in diameter.

vessel from accounting provided it is less than $1.5 \mathrm{~mm}$ in diameter.

\subsection{Overloading of the Syntax Score with Multiple Branches within Same Territory (Dx, PLV, OM)}

Cases are not uncommon where a standard size $\mathrm{Cx}$ vessel supplying the lateral LV wall has multiple OM branches of similar size $(>2-3)$ each being individually weighed with a factor of 0.5 as per the syntax scoring system (Figure 6(A)) and complex lesions like ostial stenosis or ostial occlusion in one or more of these individual branches would put an extra load on the total score which athough in reality may not have much significance as far
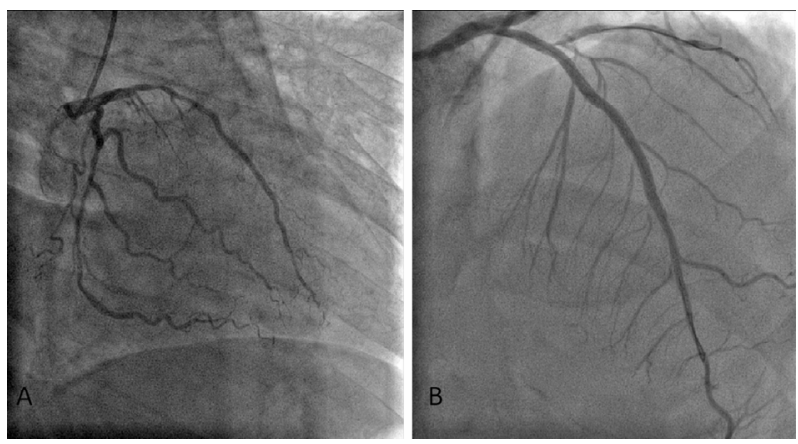

Figure 6. (A) An average sized LCX with three average sized OMs each more than $3 \mathrm{~mm}$ in diameter. An ostial lesion in all three OMs with heavily overload the SYNTAX score even though the territory supplied by these collectively remains relatively small; (B) Multiple diagonals which can overload the SYNTAX score if any of the stems had important stenosis.

as completeness and complexity of revascularization is concerned because even if this entire chunk is left untouched it might matter little as far as final out comes are concerned as these would depend more upon the proportion of entire myocardium left incompletely revascularised rather than the number of stems left non revascularised. Similar logic could stand for multiple Dx (Figure 6(B)) and multiple PLV's from RCA.

\subsection{Inappropriateness of $1.5 \mathrm{~mm}$ Calibre as the Only Criteria for a Vessel Being Considered Significant Enough to Be Accounted for}

A $1.5 \mathrm{~mm}$ diameter criteria alone may not be the most appropriate criteria for considering a vessel significant. The absolute diameter of the vessel in any patient may relate more to the total arterial texture or size of the vascular tree in general just as an individual's built or habitus. Also atherosclerosis being a generalised process, it is not uncommon in diffuse disease situations to have none of the vessels being truly $1.5 \mathrm{~mm}$ in diameter on measurement on angiogram. Does it mean that none of these vessels would be accounted for in Syntax calculations (Figure 7). The real size of a vessel or a stem is its actual myocardial value which depends upon the area of myocardium subtended by it or it is supplying in a way i.e. its myocardial weight in other terms, or so called proportion of left ventricular myocardium supplied by the individual stem irrespective of its diameter and the need for intervention must be based upon its importance thus established rather than its diameter.

\section{REMEDIES}

The Greenlane system $[10,11]$ of coronary artery reporting gives the flexibility of covering for all the anatomical variations and provides segment wise myocardial score to each individual vessel/stem which makes it an accu- 
rate and reproducible system. However, the division of myocardial segments based on this system is time consuming and tedious and requires expertise and training which would be difficult to reproduce on a computer based system. However, based on the above system we have

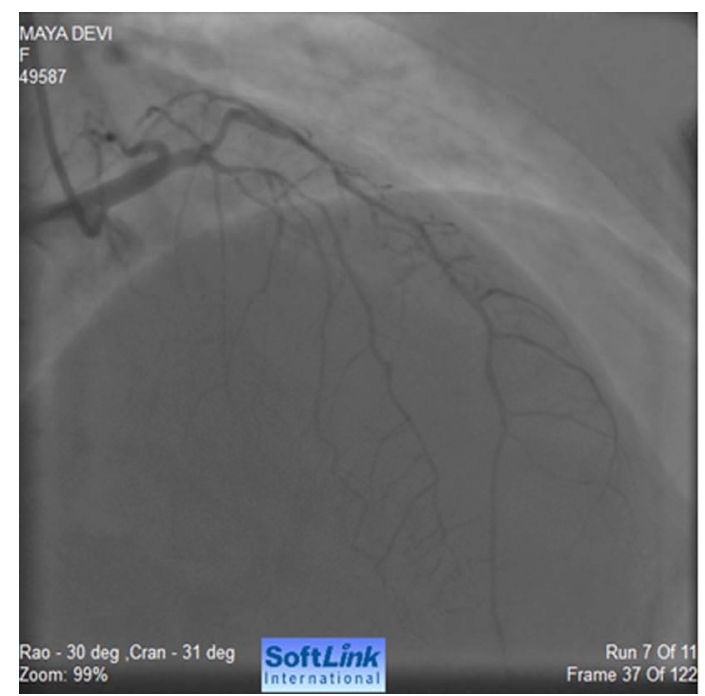

worked out a myocardial segmental scoring where in we have devised a prefixed segmental weightage, based on the basic coronary anatomy of an individual case, which takes care of most if not all of the variations and fallacies in the Syntax score highlighted by us (Table 1).

Figure 7. All vessels including LAD are of thin caibre (less than $1.5 \mathrm{~mm}$, thinner than $5 \mathrm{~F}$ catheter) but still

remain important for myocardial perfusion.

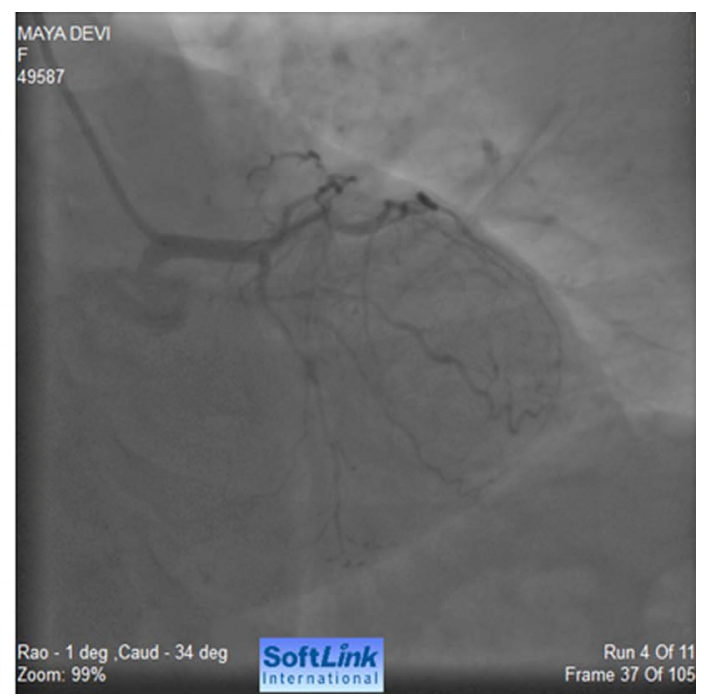

Table 1. Myocardial value of various coronary segments in different anatomical subsets of coronary anatomy.

\begin{tabular}{|c|c|c|c|c|c|c|c|c|c|c|c|c|}
\hline \multirow{2}{*}{$\begin{array}{l}\text { LAD size Vessel } \\
\text { Name }\end{array}$} & \multicolumn{3}{|c|}{ Cx. Dominant System } & \multicolumn{3}{|c|}{ Dominant RCA (PDA only) } & \multicolumn{3}{|c|}{ Dominant RCA (Average) } & \multicolumn{3}{|c|}{ Super Dominant RCA (super) } \\
\hline & Short & Average & Long & Short & Average & Long & Short & Average & Long & Short & Average & Long \\
\hline LM & 15 & 15 & 15 & 12 & 13 & 14 & 10.5 & 11.5 & 12.5 & $9(7.5)$ & $10(8.5)$ & $11(9.5)$ \\
\hline Prox. Lad & 6 & 7 & 8 & 6 & 7 & 8 & 6 & 7 & 8 & 6 & 7 & 8 \\
\hline Mid. LAD & 4 & 5 & 6 & 4 & 5 & 6 & 4 & 5 & 6 & 4 & 5 & 6 \\
\hline Distal LAD & 2 & 3 & 4 & 2 & 3 & 4 & 2 & 3 & 4 & 2 & 3 & 4 \\
\hline Major Dx & 1.5 & 1.5 & 1.5 & 1.5 & 1.5 & 1.5 & 1.5 & 1.5 & 1.5 & 1.5 & 1.5 & 1.5 \\
\hline Small Dx & 0.75 & 0.75 & 0.75 & 0.75 & 0.75 & 0.75 & 0.75 & 0.75 & 0.75 & 0.75 & 0.75 & 0.75 \\
\hline Prox. Cx & 9 & 8 & 7 & 6 & 6 & 6 & 4.5 & 4.5 & 4.5 & $3(1.5)$ & $3(1.5)$ & $3(1.5)$ \\
\hline Ramus/OM1 & 1.5 & 1.5 & 1.5 & 1.5 & 1.5 & 1.5 & 1.5 & 1.5 & 1.5 & 1.5 & 1.5 & 1.5 \\
\hline Major OM/OM2 & 1.5 & 1.5 & 1.5 & 1.5 & 1.5 & 1.5 & 1.5 & 1.5 & 1.5 & $1.5(0)$ & $1.5(0)$ & $1.5(0)$ \\
\hline Distal Cx./OM3 & 1.5 & 1.5 & 1.5 & 1.5 & 1.5 & 1.5 & 1.5 & 1.5 & 1.5 & 0 & 0 & 0 \\
\hline Distal Cx./PLV & 1.5 & 1.5 & 1.5 & 1.5 & 1.5 & 1.5 & 0 & 0 & 0 & 0 & 0 & 0 \\
\hline Prox./Mid RCA & 0 & 0 & 0 & 3 & 2 & 1 & 4.5 & 3.5 & 2.5 & $6(7.5)$ & $5(6.5)$ & $4(5.5)$ \\
\hline PDA (RCA) & 0 & 0 & 0 & 3 & 2 & 1 & 3 & 2 & 1 & 3 & 2 & 1 \\
\hline PDA (off Cx.) & 3 & 2 & 1 & 0 & 0 & 0 & 0 & 0 & 0 & 0 & 0 & 0 \\
\hline PLV 1 (RCA) & 0 & 0 & 0 & 0 & 0 & 0 & 1.5 & 1.5 & 1.5 & 1.5 & 1.5 & 1.5 \\
\hline PLV 2 (RCA) & 0 & 0 & 0 & 0 & 0 & 0 & 0 & 0 & 0 & 1.5 & 1.5 & 1.5 \\
\hline PLV 3 (RCA) & 0 & 0 & 0 & 0 & 0 & 0 & 0 & 0 & 0 & $0(1.5)$ & $0(1.5)$ & $0(1.5)$ \\
\hline $\begin{array}{l}\text { Non-Dominant } \\
\text { RCA (No PDA) }\end{array}$ & 0 & 0 & 0 & 0 & 0 & 0 & 0 & 0 & 0 & 0 & 0 & 0 \\
\hline
\end{tabular}

*Values in parenthesis relate to a situation where there are at least three major PLV's from the RCA in a super dominant right system. 
1) The issue of arterial dominance-The circulation needs to be first classified into right dominant (average), right dominant (super), left dominant (PDA from LCX) and co-dominant (PDA from RCA but PLVs from LCX) instead of right/left dominant alone. The average right dominant RCA has been given the weight of a RCA with PDA and one major PLV while standard left dominant system has entire LV supply from left circulation alone and none from RCA while a co-dominant circulation, has a RCA with myocardial weightage of only PDA from the RCA and rest of the LV supply is accomplished from $\mathrm{Cx}$. A right super-dominant system is given the myocardial weight of PDA plus $\geq 2$ PLVs for RCA and only one or two OMs from $\mathrm{Cx}$ as the case may be.

2) Similarly, a type I, II or III LAD can be pre classified and the score of the entire left tree is proportionately increased or decreased based on the length of the LAD in the system.

3) The total myocardial score in the Syntax system is six with 5.0 (84\% of LV) to LCA and $1.0(16 \%)$ to PDA while in the Greenlane system it being 15 , the different coronary segments get a proportionate score as a division of 15. A conceptual division of myocardial score for different vessel segments based on the type of circulation (Right dominant (average)/Right dominant (super)/left dominant/co-dominant) and type I/II/III LAD is shown in table attached in detail (Table 1). As an example an average RCA would contribute a score of $3.5 / 15$ with $2 / 15$ from PDA and 1.5/15 from PLV, which makes about 23\% of the LV myocardium as against $16 \%$ in the syntax system and so on.

4) The Greenlane scoring system which was formulated during the non interventional era of cardiology gives a simple understanding to intervene in any vessel when it has a myocardial value of 1.5 which in a way is a vessel supplying $10 \%$ of the entire LV myocardial supply and seems logically more correct than sticking to the 1.5 $\mathrm{mm}$ calibre criteria which has its own limitation as explained in the text above. This accounts for revascularisation of PLV/OMs/Dx done based on the size of vessel which in individual operator's mind accounts for a myocardial value of 1.5 or $10 \%$ of the entire supply of the myocardium irrespective of its anatomical diameter.

5) A complexity factor based on LV function also can be added which is the bottom line of all revascularization procedural outcomes.

\section{CONCLUSION}

We have tried to express a viewpoint on possible fallacies or limitations inherent in the syntax score and present an outline of a modified remedial system and strongly feel that a combination of the modified Greenlane system of myocardial segment weight age as suggested by us with assessment of lesion complexity as per the syntax protocol could possibly give a complexity assessment of the coronary anatomy which would be more realistic, practical and even more precise than the current syntax score without loss of ease for use. It would however need a large prospective study similar to the syntax trial to adjudicate the same and such a score would then be very close to actual decision making on PCI vs CABG in an individual patient with multivessel disease.

\section{REFERENCES}

[1] Hannan, E.L., Wu, C., Walford, G., et al. (2008) Drugeluting stents vs. coronary-artery bypass grafting in multivessel coronary disease. New England Journal of Medicine, 358, 331-341. http://dx.doi.org/10.1056/NEJMoa071804

[2] Sianos, G., Morel, M.A., Kappetein, A.P., Morice, M.C., Colombo, A., Dawkins, K., van den Brand, M., Van Dyck, N., Russell, M.E., Mohr, F.W. and Serruys, P.W. (2005) The SYNTAX Score: An angiographic tool grading the complexity of coronary artery disease. EuroIntervention, 1, 219-227.

[3] Kappetein, A.P., Feldman, T.E., Colombo, A., et al. (2011) Comparison of coronary bypass surgery with drug-eluting stenting for the treatment of left main and/or three-vessel disease: 30 -year follow-up of the SYNTAX trial. European Heart Journal, 32, 2125-2134.

http://dx.doi.org/10.1093/eurheartj/ehr213

[4] Garg, S., Sarno, G., Garcia-Garcia, H.M., et al. (2010) A new tool for the risk stratification of patients with complex coronary artery disease: The clinical SYNTAX score. Circ. Cardiovascular Interventions, 3, 317-326. http://dx.doi.org/10.1161/CIRCINTERVENTIONS.109.9 $\underline{14051}$

[5] Serruys, P.W., Unger, F., van Hout, B.A., van den Brand, M.J., van Herwerden, L.A., van Es, G.A., Bonnier, J.J., Simon, R., Cremer, J., Colombo, A., Santoli, C., Vandormael, M., Marshall, P.R., Madonna, O., Firth, B.G., Breeman, A., Morel, M.A. and Hugenholtz, P.G. (1999) The ARTS study (Arterial Revascularization Therapies Study). Seminars in Interventional Cardiology, 4, 209219.

[6] Leaman, D.M., Brower, R.W., Meester, G.T., Serruys, P. and van den Brand, M. (1981) Coronary artery atherosclerosis: Severity of the disease, severity of angina pectoris and compromised left ventricular function. Circulation, 63, 285-299. http://dx.doi.org/10.1161/01.CIR.63.2.285

[7] Hamburger, J.N., Serruys, P.W., Scabra-Gomes, R., Simon, R., Koolen, J.J., Fleck, E., Mathey, D., Sievert, H., Rutsch, W., Buchwald, A., Marco, J., Al-Kasab, S.M., Pizulli, L., Hamm, C., Corcos, T., Reifart, N., Hanrath, P. and Taeymans, Y. (1997) Recanalization of total coronary occlusions using a laser guidewire (the European TOTAL Surveillance Study). American Journal of Cardiology, 80, 1419-1423.

http://dx.doi.org/10.1016/S0002-9149(97)00704-2

[8] Topol, E.J. (1998) Textbook of interventional cardiology, 
3rd Edition, WB Saunders Co., Philadelphia, p. 728.

[9] Lefevre, T., Louvard, Y., Morice, M.C., Dumas, P., Loubeyre, C., Benslimane, A., Premchand, R.K., Guillard, N. and Piechaud, J.F. (2000) Stenting of bifurcation lesions: Classification, treatments, and results. Catheterization and Cardiovascular Interventions, 49, 274-283.

http://dx.doi.org/10.1002/(SICI)1522-726X(200003)49:3 $\leq 274:$ :AID-CCD11>3.0.CO;2-N

[10] Brandt, P.W.T., Partridge, J.B. and White, W.J. (1977)
Coronary arteriography: Methods of presentation of the arteriogram report and scoring system. Clinical Radiology, 28, 361-365.

http://dx.doi.org/10.1016/S0009-9260(77)80140-2

[11] Goel, P.K. (1997) A relook at the reporting of coronary angiograms in the interventional era: A perspective. Indian Heart Journal, 49, 323-326. 